\title{
Improving Access to Hepatitis C Treatment for Uninsured Patients in Southeast Georgia
}

\author{
Bonzo Reddick, MD, MPH ${ }^{1}$ and MariAnna O'Ree, MPH, $\mathrm{PMP}^{2}$ \\ ${ }^{1}$ Mercer University School of Medicine and ${ }^{2}$ Memorial Health University Medical Center \\ Corresponding Author: Bonzo Reddick, MD, MPH • Mercer University School of Medicine • 1250 E. 66th St., Savannah, GA 31404 • Telephone: (912) \\ 721-8221・Email: Reddick_Bi@mercer.edu
}

\section{ABSTRACT}

Background: In this brief report, we discuss enhancing access to hepatitis $\mathrm{C}$ treatment for uninsured patients in Southeast Georgia.

Methods: Through our screening program for hepatitis C virus (HCV) infections in a local safety net emergency department, the Comprehensive AIDS Resources Emergency (CARE) Initiative studied the rates of linkage to care for newly diagnosed patients with HCV and sought ways to improve linkage to care for those who did not have health insurance.

Results: Our multidisciplinary team partnered with a local federally qualified health center (FQHC) and a wholesale laboratory service to offer affordable care options, thus increasing the percent of uninsured patients who were linked to care for the treatment of their chronic HCV infections.

Conclusions: The creative use of non-invasive laboratory testing from non-traditional sources, and collaboration with pre-existing community partners is one way to make the care of chronic HCV infection attainable by uninsured and underinsured patients

Keywords: Hepatitis C, uninsured, Medicaid, CARE Initiative

\section{INTRODUCTION}

The Centers for Disease Control and Prevention (CDC), American Association for the Study of Liver Diseases (AASLD), and Infectious Diseases Society of America (IDSA) all recommend hepatitis $\mathrm{C}$ virus (HCV) screening at least once for individuals aged 18 and older (Schillie et al., 2020; AASLD and IDSA, 2019). A recent U.S. Preventive Services Task Force (USPSTF) update similarly recommends HCV testing for adults aged 18 to 79 (Chou et al., USPSTF 2019). This brief report describes how one of the Comprehensive AIDS Resources Emergency (CARE) Initiative's screening programs enhanced access to care for uninsured patients who test positive for $\mathrm{HCV}$.

\section{METHODS}

The data from this brief report come from the CARE Initiative (Okafor, 2019), a grant-funded screening program housed in the emergency department (ED) of a safety net hospital in the Savannah-Chatham County area. Safety net hospitals, as defined by the Institute of Medicine, commit to providing care to a substantial share of vulnerable patients regardless of their ability to pay (Popescu et al, 2019).
Based on the most recent national data, Georgia had the sixth highest number of newly reported cases of confirmed chronic hepatitis C out of all states reporting data in 2017 (CDC, 2019).

The CARE Initiative's HCV screening program began in February 2017, providing opt-out testing for ED patients born between 1945-1965 who were not previously tested; this was per the previous CDC guidelines (Chou et al., 2013) that recommended one-time testing only for adults born in this birth cohort and for at-risk individuals. Based on emerging evidence and the updated AASLD, IDSA, and USPSTF guidelines, we extended our screening program to include all adults aged 18 and older in March 2019. The initial screen is an $\mathrm{HCV}$ antibody test; positive antibody tests are followed by a confirmatory viral load test. Confirmed positive patients are counseled on the diagnosis by CARE linkage specialists and then linked to community providers. These linkage specialists continue with follow-up phone contact until an outpatient provider assumes care of the patient's HCV infection. 
We obtained Institutional Review Board approval to study the demographics of all patients screened for HCV through the ED to implement quality improvement and population-level interventions to decrease the burden of $\mathrm{HCV}$ infection in the community. We measured the percent of patients who were successfully linked to care for the management of their $\mathrm{HCV}$ infection and studied linkage rates for various demographic variables, including insurance status.

\section{RESULTS}

Prior to the expansion of the program, approximately 5,000 $\mathrm{HCV}$ tests were performed annually in the ED; once screening moved beyond the birth cohort to include all adults, we screened more than 15,000 patients in the subsequent year. The percentage of screened patients who tested positive $(2.7 \%)$ did not change significantly after the age inclusion criteria moved beyond the 1945-1965 birth cohort; this is likely related to the fact that many older patients returned to the ED for various medical reasons after their one-time HCV test, and they were no longer eligible for screening. The linkage rate for all patients was 39\% for the year leading up to the expansion beyond the birth cohort, and $36 \%$ in the subsequent year.

Approximately $21 \%$ of those screened for HCV in the ED were uninsured or "self-pay" patients, and we discovered significant difficulty in linking these patients to care compared to our insured patients. A physician member of the CARE Initiative also worked part-time at a local federally qualified health center (FQHC) that cares for uninsured patients, and they agreed to see patients with $\mathrm{HCV}$ there. The CARE Initiative covers the cost of the HCV tests for diagnosis and confirmation; however, FQHC administrators had concerns about their ability to cover the more than $\$ 1,000$ per patient cost of the subsequent lab testing - specifically the HCV genotype test [for the specific strain of the virus] and tests for liver cirrhosis - that are required for management after the initial diagnosis. Through online searches for wholesale laboratory services, the CARE program coordinator found a partner in January 2020 that provided an adjusted price of $\$ 300$ for the subsequent labs.

Nine uninsured patients have already been successfully linked as of the time of submission; considering our average of 85 uninsured new diagnoses annually, this trend is significant. In our city, there were previously no providers who would accept uninsured patients for management of hepatitis $\mathrm{C}$, creating a significant health inequity. Our interdisciplinary team of physicians, linkage specialists, laboratory technicians, and clinic administrators created a partnership that can be replicated to provide care for these patients.

\section{DISCUSSION/CONCLUSIONS}

Previous initiatives have demonstrated the cost-effectiveness and impact of large-scale screening programs for $\mathrm{HCV}$ in emergency departments and primary care settings (Assoumou et al, 2018; Hsieh et al., 2016). The management of our patients after diagnosis was highly variable and impacted by differences in insurance status and reimbursement. A wholesale lab company based in a different state provided affordable prices for the tests required to manage and care for our newly diagnosed, uninsured patients with $\mathrm{HCV}$. The lab service interfaces with multiple vendors and couriers that the FQHC uses for processing of results, which is how we obtained the lowest possible price.

Prior to our use of the wholesale laboratory service, we found only two providers in the county that would accept our uninsured patients diagnosed with $\mathrm{HCV}$. Because of heavy patient loads for non-HCV related issues, one of these providers agreed to accept a maximum of two patients per month, and they had strict eligibility criteria for accepting patients with $\mathrm{HCV}$-including complete sobriety from alcohol and current management of any psychiatric diagnoses. For uninsured patients that were eligible, the monthly maximum created a long wait-list.

When we approached other providers in our area about managing uninsured patients with $\mathrm{HCV}$ at perhaps a reduced rate with upfront cash payments, we encountered non-financial barriers as well. One clinic mentioned the downsides of targeting "that patient population" and had concerns about becoming known as "the Hep C Clinic." The FQHC that we ultimately partnered with is accustomed to caring for individuals of any income level and insurance status, as well as patients with psychiatric or substance abuse disorders. We found several uninsured patients willing to pay the adjusted wholesale price of $\$ 300$, and in April 2020 we were able to decrease this cost further to $\$ 180$ for most patients. Since the initial implementation has been cost neutral for the $\mathrm{FQHC}$, they are exploring the development of additional HCV screening and management programs for some of their other community partners.

\section{Acknowledgements}

The CARE Initiative would like to acknowledge JC Lewis Primary Health Care Center for its willingness to care for our patients, as well as the Patient Assistance for Laboratory Services (PALS) program for providing the discounted prices for the follow-up testing. 


\section{Disclaimer}

The CARE Initiative is funded by a FOCUS (Frontlines of Communities in the United States) grant through Gilead Sciences, Inc. FOCUS limits its support to testing and linkage to care. FOCUS does not support treatment and remains agnostic in partners' treatment decisions.

\section{References}

American Association for the Study of Liver Diseases (AASLD), Infectious Diseases Society of America (IDSA).

Recommendations for testing, managing, and treating hepatitis C. http://www.hcvguidelines.org/ (last updated November 6, 2019).

Assoumou SA, Tasillo A, Leff JA, et al. Cost-effectiveness of one-time hepatitis $\mathrm{C}$ screening strategies among adolescents and young adults in primary care settings. Clin Infect Dis 2018; 66(3): 376-84

Carlson JJ, Kowdley KV, Sullivan SD, Ramsey SD, Veenstra DL. An evaluation of the potential cost-effectiveness of non-invasive testing strategies in the diagnosis of significant liver fibrosis. J Gastroenterol Hepatol 2009; 24(5):786-91.

Centers for Disease Control and Prevention (CDC). Viral hepatitis surveillance: United States, 2017.

https://www.cdc.gov/hepatitis/statistics/2017surveillance/pdfs/2 017HepSurveillanceRpt.pdf (date released November 14, 2019).

Chou R, Cottrell EB, Wasson N, Rahman B, Guise JM. Screening for hepatitis $\mathrm{C}$ virus infection in adults: A systematic review for the U.S. Preventive Services Task Force. Ann Intern Med 2013; 158(2): 101-8.

Chou R, Dana T, Fu R, et al. Screening for hepatitis C virus infection in adolescents and adults: A systematic review update for the U.S. Preventive Services Task Force. Evidence Synthesis No. 188. AHRQ Publication No. 19-05256-EF-1. Rockville, MD: Agency for Health Research and Quality; 2019.

Hsieh YH, Rothman RE, Laeyendecker, et al. Evaluation of the Centers for Disease Control and Prevention recommendations for hepatitis $\mathrm{C}$ virus testing in an urban emergency department Clin Infect Dis. 2016;62(9):1059-65.

Okafor C. The CARE Initiative transitional care for hepatitis C patients in Savannah, GA. http://publichealth.uga.edu/wp-content/uploads/2019/10/2019-S OPH-program_final_10.18.19.pdf (last accessed May 6, 2020).

Popescu I, Fingar KR, Cutler E, Guo J, Jiang J. Comparison of 3 safety-net hospital definitions and association with hospital characteristics. JAMA Netw Open 2019; 2(8): e198577.

Schillie S, Wester C, Osborne M, Wesolowski L, Ryerson AB. $\mathrm{CDC}$ recommendations for hepatitis $\mathrm{C}$ screening among adults-United States, 2020. MMWR Recomm Rep 2020; 69(No. RR-2): 1-17.

U.S. Preventive Services Task Force (USPSTF). Hepatitis C virus infection in adults and adolescents: Screening. https://www.uspreventiveservicestaskforce.org/Page/Document/ draft-recommendation-statement/hepatitis-c-screening (last updated September 23, 2019).

(C) Bonzo Reddick and MariAnna O'Ree. Originally published in jGPHA (http://www.gapha.org/igpha/) July 24, 2020. This is an open-access article distributed under the terms of the Creative Commons Attribution Non-Commercial No-Derivatives License

(http://creativecommons.org/licenses/by/4.0/), which permits unrestricted use, distribution, and reproduction in any medium, provided the original work ("first published in the Journal of the Georgia Public Health Association...") is properly cited with original URL and bibliographic citation information. The complete bibliographic information, a link to the original publication on http://www.gapha.jgpha.org/, as well as this copyright and license information must be included. 